\title{
28 Research Square \\ Ketogenic Diet as a Potential Treatment for Traumatic Brain Injury in Mice
}

Meirav Har-Even ( $\nabla$ meiravhe@mail.tau.ac.il )

Tel Aviv University https://orcid.org/0000-0002-6294-6913

\section{Vardit Rubovitch}

Tel Aviv University

\section{Whitney A. Ratliff}

VA Healthcare System Bay Pines

\section{Bruce A. Citron}

VA New Jersey Health Care System

Chaim G. Pick

Tel Aviv University

\section{Research article}

Keywords: Ketogenic Diet, Traumatic Brain Injury, Ketone Bodies, Behavioral Tests, SIRT1, neuronal survival, Neuroinflammation

Posted Date: July 19th, 2021

DOl: https://doi.org/10.21203/rs.3.rs-642407/v1

License: (c) (i) This work is licensed under a Creative Commons Attribution 4.0 International License. Read Full License

Version of Record: A version of this preprint was published at Scientific Reports on December 1st, 2021. See the published version at https://doi.org/10.1038/s41598-021-02849-0. 


\section{Abstract}

Background: Traumatic brain injury (TBI) is a brain dysfunction without present treatment. Previous studies have shown that animals fed a ketogenic diet (KD) perform better in learning tasks than those fed a standard diet (SD) following brain injury. The goal of this study was to examine whether KD is neuroprotective in a TBI mouse model.

Methods: We utilized a closed head injury model to induce mild TBI (mTBI) in mice. Mice were fed KD or SD starting immediately following the trauma and throughout the following 30 days. Tail blood ketone bodies levels were checked at $0,3,7$ and 30 days post injury. Behavioral tests took place at 7 and 30

days post injury, visual and spatial memory impairments were assessed using the Novel object recognition (NOR) paradigm and the Y-maze test, respectively, and anxiety-like behavior was assessed using the elevated plus maze test. Primary mouse SIRT1 levels antibodies were used to detect changes in protein levels following TBI induction and treatments 7 and 30 days post injury and Immunohistochemical sections were stained with, NeuN (for mature neurons), Iba-1 (for microglia) and GFAP (for astrocyte).

Results: Elevated levels of ketone bodies were confirmed in the blood following KD. Cognitive and behavioral performance was assessed post injury and molecular and cellular changes were assessed within the temporal cortex and hippocampus. Y-maze and NOR tasks indicated that mTBI mice maintained on KD displayed better cognitive abilities than $\mathrm{mTBI}$ mice maintained on SD. Mice maintained on SD post-injury demonstrated SIRT1 reduction when compared with uninjured and KD groups. In addition, KD management attenuated mTBI-induced microglia activation and astrocyte reactivity in the dentate gyrus and decreased degeneration of neurons in the dentate gyrus and in the cortex.

Conclusion: These results support accumulating evidence that KD may be an effective approach to increase the brain's resistance to damage and suggest a potential new therapeutic strategy for treating mTBI.

\section{Background}

Traumatic Brain Injury (TBI) is a brain dysfunction that occurs as the result of an external force's impact on the brain (external impact, penetration, or rapid head movement). The most common causes of TBI's are military injuries, road accidents, falls, assaults, and sports injuries(1). Approximately 2.8 million people in the United States alone receive medical care for TBI each year, amounting to an annual cost above $\$ 76$ billion, including medical, unemployment, and additional costs $(1,2)$. Sequelae of TBI include physical, cognitive, behavioral, emotional, and social problems $(3,4)$. TBI results in many pathophysiological changes that develop in two phases. Primary brain injury, which is a direct consequence of the external force exerted on the brain, consists of tissue distortion and destruction proximal to the injury and disruption of axons and small vessels, causing immediate necrotic neuronal cell death(5). Processes initiated during the primary phase lead to progressive and extended secondary 
damage, including neuroinflammation, oxidative stress, and glutamate excitotoxicity, which contribute to neuronal apoptotic cell death $(5,6)$. Mild TBI (mTBI), which accounts for over $80-90 \%$ of all TBI cases, is difficult to diagnose because routine tests, including imaging, fail to show changes in brain structure $(7,8)$. Despite this, mTBI patients frequently suffer short and long-lasting cognitive, behavioral, and emotional impairments(9). Such impairments include, among others, memory and concentration deficits, poor executive functions, depression, and anxiety-related disorders(10).

The ketogenic diet (KD) is a high-fat, low carbohydrate diet, originally designed to stimulate the beneficial biochemical changes found in the fasting state, and has been used in patients with difficult-to-treat epilepsy since 1921(11). KD is one method for inducing ketosis, a metabolic state in which the body uses ketone bodies as energy supply instead of glucose, the primary substrate for energy metabolism in the body and brain. Recent studies have begun to explore ketogenic therapies in other neurological and psychiatric disorders, including Parkinson's disease(12) and Alzheimer's disease(13, 14). An increasing number of murine studies suggest that KD induces anti-inflammatory effects(15). Studies have reported that KD, and in particular, the ketone metabolite beta-hydroxybutyrate suppresses activation of the NLRP3 inflammation in response to several structurally unrelated NLRP3 activators(15) and improves the brain's ischemic tolerance(16). KD management has also been shown to reduce activated microglial expression(17). While mechanism of KD in neuroprotection is unknown, past reports found that KD increases glutathione level(18) and Uncoupling protein (UCP)(19) in cells after brain injuries, decreasing Reactive Oxygen Species (ROS).

A possible mechanism by which KD could be inducing neuroprotective effects is through the alteration of SIRT1 expression. SIRT1 has a role in developing the hippocampus by activating Akt and inhibiting GSK3 and is involved in various physiological processes such as oxidative stress response, genetic silencing, genome stability, and cell life extension $(20,21)$. Studies have shown that the Sirtuin family of proteins is involved in the hypothalamus, where it plays roles in regulating the circadian cycle, endocrine pathways, and appetite(22-25). Moreover, high fat diet and increased circulating ketone bodies have been shown to activate SIRT1(26).

In the present study, we utilize a closed head weight drop model of murine mTBI to test the cognitive, cellular, and molecular effects of up to 30 days of KD management following injury. We report that KD initiated after $\mathrm{mTBI}$ ameliorated the cognitive deficits in spatial and visual memory, as well as cellular changes in neurons and glial cells induced by the injury. Our model also shows that KD sustained the levels of SIRT1 expression which were decreased with injury.

\section{Methods}

\section{Experimental Procedures}

Mice were subjected to $\mathrm{mTBl}$, and a ketogenic diet (KD) was initiated immediately for the following three timelines: 3 days, 7 days, and 30 days. Ketone bodies in the blood were measured $0,3,7$, and 30 days 
post-injury. Behavioral tests were performed at 7 or 30 days following mTBI in separate groups of animals. Western blot analysis was carried out on brain tissue collected from 7 and 30 days post mTBI. Immunohistochemical staining assessments were performed on brains that were collected 30 days postinjury. The timeline of the experimental procedures following exposure to mTBI is shown in Fig. 1.

\section{Animals}

The Sackler Commission on Animal Experimentation approved the animal protocol 01-19-058 according to the Guidelines for Animal Experimentation of the National Institutes of Health (DHEW publication 2385 , revised, 1995).

Male ICR mice, aged 6-7 weeks, 30 to $40 \mathrm{~g}$ body weight, were acquired from Envigo RMS Israel. Mice were housed at $4-5$ per home cage under a constant $12 \mathrm{~h}$ light/dark cycle, at room temperature $\left(22 \pm 2^{\circ} \mathrm{C}\right)$ in a standard plastic cage $\left(32 \times 21.5 \times 12 \mathrm{~cm}^{3}\right)$. Water and food was provided ad libitum, and the cages bedding, sterile sawdust, was replaced once per week. All mice were acclimatized to the facility for three days following transport and then moved into the experimental testing room for three days prior to experimentation. Animals were utilized only once throughout the study in either behavioral, biochemistry, or immunocytochemistry tests. The number of animals evaluated in each assessment group and the measurement times performed was based on analysis of variance in our previous studies.

\section{Mouse closed-head mild traumatic brain injury}

Mild Traumatic Brain Injury (mTBI) was induced according to the closed-head weight-drop model, as employed in our previous studies $(27,28)$. The device consisted of an aluminum tube $(80 \mathrm{~cm}$ in length and $13 \mathrm{~mm}$ in diameter). In the pre-injury stage, mice were anesthetized by inhalation of isoflurane and placed under the device on a sponge to allow for rotation of the head upon impact. A metal weight $(50 \mathrm{~g})$ was dropped from the top of the tube to strike the head on the right temporal side between the ear and corner of the eye. Sham mice received anesthesia and were placed on the sponge for an equivalent length of time, but no weight was dropped. This model was chosen because it simulates traumatic head injuries such as road accidents or falls, as it imposes a diffuse and non-specific injury(27-30).

\section{Ketogenic Diet}

The macronutrient composition of the Ketogenic Diet (TD.96355; Harlan Teklad Laboratories, Madison, WI) was $9.2 \%$ protein, $0.3 \%$ carbohydrate, and $90.5 \%$ fat (\% kcal) (Table 1$)(31-33)$. The corresponding components of the standard diet (SD) (TD.00606; Harlan Teklad Laboratories, Madison, WI) were 10.1\% protein, $77.4 \%$ carbohydrate, and $12.5 \%$ fat (\% kcal)(31). The diets and water were provided ad libitum for 3,7 , and 30 days after the injury. Both diets were stored at $4^{\circ} \mathrm{C}, \mathrm{KD}$, which had a solid, butter-like texture, was changed every day, and SD pellets were changed twice per week. 
Table 1

Macronutrient information of each diet

\begin{tabular}{|lll|}
\hline \% Kcal from & Standard Diet & Ketogenic Diet \\
\hline Fat & 12.5 & 90.5 \\
\hline Carbohydrate & 77.4 & 0.3 \\
\hline Protein & 10.1 & 9.2 \\
\hline
\end{tabular}

\section{Measurement of Ketone Bodies}

Ketone bodies in the blood were measured using the Precision Xtra Blood Glucose and Ketone Monitoring System (Abbott, Columbus, $\mathrm{OH}$ (34). Mice were anesthetized with isoflurane and tails were cut approximately $0.2 \mathrm{~mm}$ from the end, a drop of blood was squeezed directly into a testing strip attached to the measuring instrument.

\section{Elevated Plus Maze}

The elevated plus maze (EPM) was used to evaluate anxiety-like behavior(35). This assessment relies on the natural anxiety-like behavior exhibited by rodents when placed in brightly lit, open environments. The maze is plus-shaped (+), with arms extending from the center at $90^{\circ}$ angles from each other. Opposite arms in the plus formation are identical, with two open arms and two closed (walled) arms measuring 30 $x 5 \times 1 \mathrm{~cm}$ each. During testing, each mouse was placed at the maze's center, facing one of the open arms, and was allowed to explore the maze for 5 minutes. The amount of time the mice spent in the open arms and the number of entries to the closed and open arms was counted. A longer duration of time spent within the open arms has been associated with lower anxiety levels(35, 36).

\section{Novel Object Recognition}

The novel object recognition (NOR) task assesses recognition and visual memory(37). This paradigm relies on rodents' natural tendency to investigate novel objects within their environment rather than known ones. NOR evaluates whether a mouse is able to discriminate between a familiar and a novel object. The testing arena is a square surface $(60 \mathrm{~cm} \times 60 \mathrm{~cm})$ with high walls $(20 \mathrm{~cm})$. The test consists of three 5 min sessions, separated by 24 hours. On the first day, mice were individually put in the empty arena for habituation for 5 minutes. On the second day, the mice were exposed to 2 identical objects within the arena for 5 minutes. On the third (experimental) day, one of the familiar objects was replaced with a novel object, and mice were allowed to explore the arena again for 5 minutes, during which time spent near familiar and novel object was measured. The arena was cleaned with $70 \%$ ethanol between subjects. An Aggelton index was calculated as follows: (time near new novel object - time near familiar object)/(time near new novel object + time near familiar object)(38). A higher Aggelton index indicates advancement in recognition memory. Animals near the objects less than $10 \%$ of the total test time (i.e., less than 30 s next to the two objects together) were excluded from statistical calculations, as exploration under this amount of time does not allow estimation of subjects' visual memory. 


\section{Y-maze}

The Y-maze paradigm was used to evaluate spontaneous exploration, responsiveness to novel environments, and spatial memory function, as previously described(39). This test relies on the preference of rodents to explore new environments rather than familiar ones. The Y-maze consists of a three-armed black plexiglass maze with arms separated by $120^{\circ}$. Each arm was identical $(8 \times 30 \times 15$ $\mathrm{cm}$ ); however, different spatial cues were placed in each arm (i.e., a triangle, a square, or a circle). In the first session of the test, the mouse was put in the arena's start arm (chosen randomly) and allowed to explore another arm while the third arm was blocked for 5 minutes. The mouse was then returned to its home cage for 2 minutes. In the second session, all arms were open for exploration for 2 minutes and time spent in each arm was recorded. The arena was cleaned with $70 \%$ ethanol between subjects. The time the mouse spent in the familiar arm and the new arm was measured. An Aggelton index was calculated as follows: (time in the new arm - time in the familiar arm)/(time in new arm + time in the familiar arm)(38). A higher Aggelton index indicates improved spatial memory.

\section{Immunohistochemistry}

Immunohistochemistry studies were performed hippocampal (dentate gyrus) and temporal cortex tissue sections obtained from animals euthanized on day 30 post-injury. Mice were anesthetized with ketamine $(100 \mathrm{mg} / \mathrm{kg})$ and xylazine $(10 \mathrm{mg} / \mathrm{kg})$ and underwent transcardial perfusion with $10 \mathrm{ml}$ phosphatebuffered saline (PBS) followed by $20 \mathrm{ml}$ of $4 \%$ paraformaldehyde (PFA) in $0.1 \mathrm{M}$ phosphate buffer, $\mathrm{pH}$ 7.4. Brains were removed, fixed overnight in $4 \%$ PFA, and then placed in 1\% PFA. Neuroscience Associates (Knoxville, TN) oriented the brains into a multiblock, collected $35 \mu \mathrm{m}$ sections sequentially through the brains, and performed the floating section staining and mounting (antibodies detailed in Table 2). Microscopy was performed with a Fluoview 3000 laser scanning confocal microscope (Olympus, Waltham, MA). Target locations were determined on a stitched map with only Hoechst 33342 staining captured. Images, centered on coronal sections at approximately $-2.9 \mathrm{~mm}$ from Bregma, were collected as $Z$ stacks and maximum $Z$ projections with constant illumination $(405,488,561$, and $640 \mathrm{~nm}$ diode lasers) and detection parameters. Automated analysis for morphology, intensities, and numbers of cells was conducted using cellSens (Olympus, Waltham, MA) and Image $(39,40)$. Frames included in figures are correct concerning orientation, i.e., dorsal at the top, left, and right. Confocal scanning was rotated $30^{\circ}$ to optimize the framing of regions of interest. 
Table 2

Immunohistochemistry reagents

\begin{tabular}{|c|c|c|c|c|c|}
\hline $\begin{array}{l}\text { Target / } \\
\text { Fluorochrome }\end{array}$ & $\begin{array}{l}\text { Primary / } \\
\text { Secondary }\end{array}$ & Probe & Manufacturer & Catalog & Dilution \\
\hline Nuclei & & Hoechst 33342 & ThermoFisher & & \\
\hline Astrocytes & Primary & $\begin{array}{l}\text { Chicken anti- } \\
\text { GFAP }\end{array}$ & Encor & CPCA-GFA & $1: 1500$ \\
\hline Alexa488 & Secondary & $\begin{array}{l}\text { Donkey anti- } \\
\text { chicken }\end{array}$ & Jackson & $\begin{array}{l}703-545- \\
155\end{array}$ & $1: 500$ \\
\hline Microglia & Primary & Goat anti-lba1 & ThermoFisher & $\begin{array}{l}\text { PA5- } \\
18039\end{array}$ & $1: 1500$ \\
\hline Alexa555 & Secondary & $\begin{array}{l}\text { Donkey anti- } \\
\text { goat }\end{array}$ & ThermoFisher & A21432 & $1: 500$ \\
\hline NeuN + Neurons & Primary & $\begin{array}{l}\text { Rabbit anti- } \\
\text { NeuN }\end{array}$ & Abcam & Ab104225 & $1: 5000$ \\
\hline Alexa647 & Secondary & $\begin{array}{l}\text { Donkey anti- } \\
\text { rabbit }\end{array}$ & ThermoFisher & A31573 & $1: 500$ \\
\hline
\end{tabular}

\section{Western Blotting}

To assess the cortical and hippocampal SIRT1 levels, brains were dissected following cervical dislocation at 7 and 30 days post-injury. The right/left cortex and right/left hippocampus were separated and frozen in liquid nitrogen, then stored in $-80^{\circ} \mathrm{C}$. Prior to analysis, brains were homogenized in lysis buffer (Tissue Protein Extraction Reagent, Pierce, Waltham, MA) supplemented with a protease inhibitor cocktail (Halt Protease Inhibitor Cocktail, Sigma Aldrich, St. Louis, MO) using a Teflon pestle homogenizer.

Homogenates were centrifuged for 15 minutes at $4^{\circ} \mathrm{C} 14,000 \mathrm{r} / \mathrm{min}$, supernatant liquids were separated from the precipitates and stored at $-80^{\circ} \mathrm{C}$. Sample buffer was added to the samples and then stored at $-18^{\circ} \mathrm{C}$. Prior to analysis, samples were heated to $90^{\circ} \mathrm{C}$ for 3 minutes and $30 \mu$ l of each sample was then loaded and run on 4-20\% Mini-Protean TGX gels (Bio-Rad, Hercules, CA) followed by transfer onto nitrocellulose membranes (Bio-Rad, Hercules, CA) by a transfer system (Trans-Blot Turbo, Bio-Rad, Hercules, CA). Afterward, blots were blocked for 1 hour at room temperature, with Tris-buffered saline, containing $0.01 \%$ Tween-20 and 5\% BSA or powdered milk. Membranes were then incubated overnight at $4^{\circ} \mathrm{C}$ with a mouse primary anti-SIRT1 antibody (Abcam, Cambridge, UK, ab10304, 1:500) and washed with TBS. Membranes were then incubated at room temperature for 1 hour with goat anti-mouse antibody (Jackson ImmunoResearch Laboratories, Inc., West Grove, PA, 115-035-003, 1:10,000). Bands were then exposed using enhanced chemiluminescence with ECL (Millipore, Billerica, MA, United States) for 1 minute by Viber Fusion FX7 imaging system (Viber Lourmat, France). Densitometry analysis of the detected signal was performed using ImageJ software. Uniform loading was verified by stripping and reprobing with a mouse primary a-tubulin antibody for 30 minutes at room temperature (Santa Cruz Biotechnology, Dallas, TX, sc-53030, 1:10,000), then conjugated goat anti-mouse antibody (Jackson 
ImmunoResearch Laboratories, Inc., West Grove, PA, 115-035-003, 1:10,000). The ratio of SIRT1 and atubulin determined the value of each sample. Averages of control values in each membrane were set to 1 , and all other samples were calculated accordingly(41).

\section{Statistical Analysis}

All values are presented as mean values \pm standard error (SEM). Significance was calculated using the ANOVA (analysis of variance) tests for continuous variables. For post hoc comparisons, we used Fisher LSD post hoc tests. One or Two-way ANOVA was performed in SPSS 24 (IBM, Armonk, NY). Statistically significant differences between the averages will be marked by asterisk, $* \mathrm{p}<0.05, * * \mathrm{p}<0.01, * * * \mathrm{p}<$ 0.001 .

\section{Results}

$K D$ increased the level of ketone bodies in blood

To confirm whether KD increased the levels of ketone bodies in the blood, we used a ketone monitoring system at various time points. Mice placed on KD (with or without $\mathrm{mTBI}$ ) demonstrated a prominent increase in ketone bodies compared to mice on SD at 3, 7, and 30 days after the diet was initiated (Fig. 2).

$m T B I$ exposure and KD management does not affect anxiety

Following $\mathrm{mTBI}$ and diet management, male ICR mice were evaluated for anxiety-like behavior using the elevated plus maze (EPM) at 7 and 30 days post-injury. The anxiety-like behavior was estimated by analyzing the time spent in the open arms of the maze. There were no differences in time spent in the open arms between all groups at both time points tested, indicating that the mice's anxiety-like behavior was not affected either by the injury or diet management (data not shown).

\section{$K D$ ameliorates cognitive deficits}

NOR and Y-maze paradigms were performed on mice at 7 and 30 days post-injury to assess the effects of KD on visual and spatial memory following injury. During NOR testing, time spent investigating the novel/familiar object or arm was analyzed to evaluate visual memory. mTBI-challenged mice suffered a significant visual memory deficit, as evidenced by reduced novel object exploration time compared to sham mice at both 7 and 30 days post-injury $(p<0.001$, Fig. 3A-B). This deficit was substantially ameliorated by KD management at both 7 and 30 days post-injury ( $p<0.001$ and $p<0.01$ respectively). The Y-maze paradigm was implemented to evaluate spatial memory. At both time points evaluated, mTBI-challenged mice experienced spatial memory impairment compared to sham mice $(p<0.001$, Fig. 3C-D). Although spatial memory of TBI + KD mice at 7 days did not improve to the levels seen in the control mice (Fig .3C), as with the NOR test, KD-treated mTBI mice demonstrated significantly higher new arm discriminatory preference than mTBI mice on SD at 7 and 30 days post-injury $(p<0.01)$. 
Levels of SIRT1 were significantly reduced 30 days post-injury in the cortex (right and left) of mTBI mice compared with control $(p<0.05)$ and 30 days post-injury in the right (ipsilateral to injury) hippocampus ( $p$ $<0.01$ ) (Fig. 4A-D). The KD prevented this reduction in the right cortex $(p<0.01)$, in the left cortex $(0.05)$, and in the right hippocampus $(p<0.001)$. However, SIRT1 levels were not significantly reduced in the left (contralateral to injury) hippocampus of mice compared with control mice. In contrast, 7 days post-injury SIRT1 levels were not significantly reduced in the cortex and hippocampus compared with control mice (data non shown).

Ketogenic Diet prevents mTBI-induced neuronal loss

Neuronal loss induced by mTBI was evaluated immunohistochemically in the dentate gyrus and temporal cortex using a NeuN antibody at 30 days post-injury. The NeuN is a DNA-binding neuron-specific protein which can be used to visualize mature neurons via immunohistochemical staining. In this experiment, $\mathrm{mTBI}$ challenge in the absence of KD resulted in a significant decrease in neuronal survival of approximately $15 \%$, when compared to all other groups (Fig. 5). This cellular loss was diffuse and occurred throughout both of the evaluated brain regions.

Ketogenic Diet mitigates TBI-induced neuroinflammation by reducing activated microglia and reactive astrocytes

Changes in glial cells occurring subsequent to mTBI was evaluated 30 days post-injury in the dentate gyrus and temporal cortex through staining of astrocytes by GFAP and microglia by Iba-1. mTBI induced a significant elevation in astrocyte and microglial reactivity in the dentate gyrus hillus (DGH) ${ }^{*} p=0.0146$ and ${ }^{*} p=0.0276$ respectively) but not in the temporal cortex (Fig. 6A-B, D). Astrocytes in the DGH region displayed approximately twice the ramification morphology as the control samples $\left({ }^{*} P=0.01\right)$ reflective of reactive astrocytosis (Fig. $6 \mathrm{C}$ ). There were no significant differences in the temporal cortex.

\section{Discussion}

TBI is a leading cause of death and long-term disability in the developed world, with more than 10 million people suffering worldwide every year(42). The majority of these TBls (80 to $95 \%)$ are mild in nature( 7,8$)$. TBI symptoms can occasionally resolve within the first year after injury, but up to $70-90 \%$ of patients continue to manifest prolonged and often permanent neurocognitive dysfunction. In light of the growing reports suggesting beneficial effects of KD in many neurological disorders (12-14), the primary goal of this study was to assess the benefits of KD in $\mathrm{mTBI}$. Our results suggest that KD may be a vital treatment modality, mitigating TBI-induced cognitive impairments, neuronal loss, and neuroinflammation in the closed-head mTBI mouse model.

We delivered a mild TBI to mice followed by up to 30 days of KD or SD. We were able to confirm that mice who received $\mathrm{KD}$ (with or without $\mathrm{mTBI}$ ) demonstrated a prominent increase in ketone bodies compared to mice fed SD at 3, 7, and 30 days after the diet. Previous studies have demonstrated that mice challenged with mTBI show cognitive impairments in visual and spatial memory $(10,28)$. Our previous 
published studies as well as the present study, ruled out the possible involvement of anxiety (as assessed by EPM) in the cognitive performance of the injured mice. This study's results replicated the previous findings regarding visual and spatial memories while demonstrating that KD significantly ameliorated these cognitive deficits. These results support previous studies on the benefits of KD on brain injury in the short-term (7d); rats who were fed KD exhibited evidence of neuroprotection after head trauma in the cortex and hippocampus(29) and improvement in motor performance in Beam balance and Beam walk tests(17).

To better understand the molecular effects of TBI and KD, we evaluated SIRT1 levels in four brain regions ipsilateral (right) cortex, contralateral (left) cortex, ipsilateral hippocampus, and contralateral hippocampus which play crucial roles in memory formation. TBI significantly reduced the levels of SIRT1 in cortex and in the ipsilateral hippocampus 30 days post-injury which was ameliorated by KD, suggesting a potential mechanism contributing to cognitive impairment and improvement in cognitive symptoms when given $\mathrm{KD}(45)$. In contrast, at 7 days post-injury SIRT1 levels were not reduced in the cortex and hippocampus. This supports past findings showing an elevation in SIRT1 levels 1 day prior to ischemia that gradually decreased over a period of 7 days(46). Our study's results align with prior studies of intermittent fasting and caloric restriction for 30 days post-injury(41) and with research involving KD and SIRT1 in health-enhancing, aging, longevity, and neurodegeneration in animal models $(33,34)$.

We have previously reported that our mTBI model decreases the neuronal survival 3 weeks post injury(49). Similarly, in the present study, we have shown a significant increase in neuronal cell death that persists 30 days following injury in both cortex and hippocampus. KD prevented this neuronal cell death. These results are consistent with an in vitro study that found the ketone body beta-hydroxybutyrate reduced axonal degeneration in diffuse axonal injury(50). Additionally, we saw a marked increase in activated microglia and reactive astrocytes in hippocampus 30 days following injury, which was ameliorated by KD. We have previously reported that our $\mathrm{MTBI}$ model induces fundamental neuroinflammatory changes, including elevations in astrocyte and microglial reactivity, pro-inflammatory cytokine TNF-a levels, and expression of genes involved in inflammatory processes in several regions of the brain $(37-40,45)$. We can conclude that KD prevented the injury-induced neuro-inflammation, which is in agreement with previous studies that reported KD inhibited NLRP3 inflammasome activation, thus exerting neuroprotective effects(16).

\section{Conclusion}

Our closed head injury mouse model of mild TBI was able to recapitulate the cognitive deficits observed in human TBI patients $(51,52)$ and showed that ketogenic diet beginning following injury could protect against injury-induced memory loss. At the cellular level, we have demonstrated that mTBI induced neuronal cell death, astrocyte and microglial activation (neuro-inflammation), which were prevented in injured mice treated with KD. Our analysis of changes in SIRT1 suggests a viable molecular mechanism by which KD may be improving cognitive outcomes. Our results support accumulating evidence that KD 
may represent an important non-pharmaceutical treatment against the long-term molecular and cellular impacts of mTBI, which may ultimately improve cognitive symptoms and quality of life for TBI patients.

\section{List Of Abbreviations}

Akt - Protein Kinase B

ANOVA - Analysis of variance

EPM - Elevated plus maze

GSK3 - Glycogen synthase kinase 3

KD - Ketogenic diet

mTBI - Mild traumatic brain injury

NLRP3 - NLR family pyrin domain containing 3

NOR - Novel object recognition

NS- Non-significant

ROS - Reactive oxygen species

SD - Standard diet

SIRT 1 - Sirtuin 1

TBI - Traumatic brain injury

TNF-a - Tumor necrosis factor alpha

UCP - Uncoupling protein

\section{Declarations}

\section{Ethical Approval and Consent to participate}

The Sackler Commission on Animal Experimentation approved the animal protocol 01-19-058 according to the Guidelines for Animal Experimentation of the National Institutes of Health (DHEW publication 2385, revised, 1995).

Consent to participate was not applicable.

Consent for publication

Not applicable 
Availability of supporting data

All data supporting this study and its findings are available within the article or from the corresponding author upon reasonable request.

\section{Competing Interests}

No competing financial interests exist.

\section{Funding}

This research was funded by the Ari and Regine AprijaskisFund, grant number 347300-00; the Dr. Miriam and Sheldon G. Adelson Center for the Biology of Addictive Diseases, grant number 601133461; and the Sylvan Adams Sports Institute grant number 0601133671.

\section{Author Contributions}

M.H.-E, V.R. and C.G.P designed the experiment. M.H.-E. and B.A.C performed the experiments and analysed the data. V.R. and W.A.R gave technical support and conceptual advice. B.A.C supervised the analysis. M.H.-E wrote the manuscript. W.A.R, V.R. and C.G.P edited the manuscript.

All authors read and approved the final manuscript.

\section{Acknowledgements}

This research was supported in part by the Ari and Regine Aprijaskis Fund at Tel-Aviv University and the Dr. Miriam and Sheldon G. Adelson Chair for the Biology of Addictive Diseases in Tel-Aviv University, TelAviv, Israel. The contents do not represent the views of the Department of Veterans Affairs or the United States Government and the opinions, interpretations, conclusions and recommendations are those of the authors and are not necessarily endorsed by the Department of Defense.

\section{Author Information}

Meirav Har-Even, RD, M.Sc., Tel Aviv University, Department of Anatomy and Anthropology, Sackler School of Medicine Building, Room 624a; Tel Aviv 6997801, Israel; Tel: +972 504-745158; meiravhe@mail.tau.ac.il

Vardit Rubovitch, Ph.D., Tel Aviv University, Department of Anatomy and Anthropology, Sackler School of Medicine Building, Room 624a; Tel Aviv 6997801, Israel; Tel: +972 507-476014; rubovitc@tauex.tau.ac.il

Whitney A. Ratliff, Ph.D., 10000 Bay Pines Blvd., Bay Pines VA Healthcare System, Laboratory of Molecular Biology, Research and Development 151, Bldg. 22, Rm. 123 R\&D 151, Bay Pines, FL 337444125, Tel: +1 727-398-6661 x14171; fax: +1 727-319-1161; whitney.ratliff@va.gov 
Bruce A. Citron, Ph.D., 385 Tremont Ave., VA New Jersey Health Care System, Laboratory of Molecular Biology, Research and Development 151, Building 16, Room 176, East Orange, NJ 07018 Tel: +1 973-6761000 x3686; bruce.citron@rutgers.edu

Chaim G., Pick, Ph.D., Tel Aviv University, Department of Anatomy and Anthropology, Sackler School of Medicine Building, Room 633; Tel Aviv 6997801, Israel; Tel: +972 544-320012; fax: +972 3-6408287; pickc@tauex.tau.ac.il

\section{References}

1. Taylor CA, Bell JM, Breiding MJ, Xu L. Traumatic Brain Injury-Related Emergency Department Visits, Hospitalizations, and Deaths - United States, 2007 and 2013. MMWR Surveill Summ. 2017;66(9):116.

2. Centers for Disease Control and Prevention (CDC). CDC grand rounds: reducing severe traumatic brain injury in the United States. MMWR Morb Mortal Wkly Rep [Internet]. 2013 Jul 12;62(27):54952. Available from: http://www.ncbi.nlm.nih.gov/pubmed/23842444.

3. Rabinowitz AR, Levin HS. Cognitive sequelae of traumatic brain injury. Psychiatr Clin North Am. 2014 Mar;37(1):1-11.

4. Rao V, Lyketsos C. Neuropsychiatric sequelae of traumatic brain injury. Psychosomatics. 41(2):95103.

5. Greve MW, Zink BJ. Pathophysiology of traumatic brain injury. Mt Sinai J Med [Internet]. 2009 Apr;76(2):97-104. Available from: http://www.ncbi.nlm.nih.gov/pubmed/19306379.

6. Werner C, Engelhard K. Pathophysiology of traumatic brain injury. Br J Anaesth. 2007 Jul;99(1):4-9.

7. De Kruijk JR, Twijnstra A, Leffers P. Diagnostic criteria and differential diagnosis of mild traumatic brain injury. Brain Inj. 2001 Feb;15(2):99-106.

8. Tashlykov V, Katz Y, Volkov A, Gazit V, Schreiber S, Zohar O, et al. Minimal traumatic brain injury induce apoptotic cell death in mice. J Mol Neurosci [Internet]. 2009 Jan;37(1):16-24. Available from: http://www.ncbi.nIm.nih.gov/pubmed/18651249.

9. Daneshvar DH, Riley DO, Nowinski CJ, McKee AC, Stern RA, Cantu RC. Long-Term Consequences: Effects on Normal Development Profile After Concussion. Phys Med Rehabil Clin N Am. 2011 Nov;22(4):683-700.

10. Li Y, Bader M, Tamargo I, Rubovitch V, Tweedie D, Pick CG, et al. Liraglutide is neurotrophic and neuroprotective in neuronal cultures and mitigates mild traumatic brain injury in mice. J Neurochem. 2015 Dec;135(6):1203-17.

11. Wheless JW. History of the ketogenic diet. Epilepsia [Internet]. 2008 Nov;49 Suppl 8:3-5. Available from: http://www.ncbi.nlm.nih.gov/pubmed/19049574.

12. Phillips MCL, Murtagh DKJ, Gilbertson LJ, Asztely FJS, Lynch CDP. Low-fat versus ketogenic diet in Parkinson's disease: A pilot randomized controlled trial. Mov Disord. 2018;33(8):1306-14. 
13. Vanltallie TB. Biomarkers, ketone bodies, and the prevention of Alzheimer's disease. Metabolism. 2015 Mar;64(3 Suppl 1):51-7.

14. Hertz L, Chen Y, Waagepetersen HS. Effects of ketone bodies in Alzheimer's disease in relation to neural hypometabolism, $\beta$-amyloid toxicity, and astrocyte function. J Neurochem. 2015 Jul;134(1):720.

15. Youm YH, Nguyen KY, Grant RW, Goldberg EL, Bodogai M, Kim D, et al. The ketone metabolite $\beta$ hydroxybutyrate blocks NLRP3 inflammasome-mediated inflammatory disease. Nat Med. 2015.

16. Guo M, Wang X, Zhao Y, Yang Q, Ding H, Dong Q, et al. Ketogenic Diet Improves Brain Ischemic Tolerance and Inhibits NLRP3 Inflammasome Activation by Preventing Drp1-Mediated Mitochondrial Fission and Endoplasmic Reticulum Stress. Front Mol Neurosci [Internet]. 2018;11:86. Available from: http://www.ncbi.nlm.nih.gov/pubmed/29662437.

17. Zhang F, Wu H, Jin Y, Zhang X. Proton Magnetic Resonance Spectroscopy (H1-MRS) Study of the Ketogenic Diet on Repetitive Mild Traumatic Brain Injury in Adolescent Rats and Its Effect on Neurodegeneration. World Neurosurg. 2018.

18. Jarrett SG, Milder JB, Liang L-P, Patel M. The ketogenic diet increases mitochondrial glutathione levels. J Neurochem [Internet]. 2008 Aug;106(3):1044-51. Available from: http://www.ncbi.nlm.nih.gov/pubmed/18466343.

19. Sullivan PG, Rippy NA, Dorenbos K, Concepcion RC, Agarwal AK, Rho JM. The ketogenic diet increases mitochondrial uncoupling protein levels and activity. Ann Neurol [Internet]. 2004 Apr;55(4):576-80. Available from: http://www.ncbi.nlm.nih.gov/pubmed/15048898.

20. Martin A, Tegla CA, Cudrici CD, Kruszewski AM, Azimzadeh P, Boodhoo D, et al. Role of SIRT1 in autoimmune demyelination and neurodegeneration. Immunol Res [Internet]. 2015 Mar;61(3):187-97. Available from: http://www.ncbi.nlm.nih.gov/pubmed/25281273.

21. Pasinetti GM, Wang J, Ho L, Zhao W, Dubner L. Roles of resveratrol and other grape-derived polyphenols in Alzheimer's disease prevention and treatment. Biochim Biophys Acta. 2015 Jun;1852(6):1202-8.

22. Sasaki T, Kim H-J, Kobayashi M, Kitamura Y-I, Yokota-Hashimoto H, Shiuchi T, et al. Induction of hypothalamic Sirt1 leads to cessation of feeding via agouti-related peptide. Endocrinology. 2010 Jun;151(6):2556-66.

23. Ramadori G, Fujikawa T, Anderson J, Berglund ED, Frazao R, Michán S, et al. SIRT1 deacetylase in SF1 neurons protects against metabolic imbalance. Cell Metab. 2011 Sep;14(3):301-12.

24. Cohen DE, Supinski AM, Bonkowski MS, Donmez G, Guarente LP. Neuronal SIRT1 regulates endocrine and behavioral responses to calorie restriction. Genes Dev. 2009 Dec;23(24):2812-7.

25. Chang H-C, Guarente L. SIRT1 mediates central circadian control in the SCN by a mechanism that decays with aging. Cell. 2013 Jun;153(7):1448-60.

26. Scheibye-Knudsen M, Mitchell SJ, Fang EF, lyama T, Ward T, Wang J, et al. A high-fat diet and NAD(+) activate Sirt1 to rescue premature aging in cockayne syndrome. Cell Metab [Internet]. 2014 Nov 4;20(5):840-55. Available from: http://www.ncbi.nlm.nih.gov/pubmed/25440059. 
27. Zohar O, Schreiber S, Getslev V, Schwartz JP, Mullins PG, Pick CG. Closed-head minimal traumatic brain injury produces long-term cognitive deficits in mice. Neuroscience [Internet]. 2003;118(4):94955. Available from: http://www.ncbi.nlm.nih.gov/pubmed/12732240.

28. Baratz R, Tweedie D, Rubovitch V, Luo W, Yoon JS, Hoffer BJ, et al. Tumor necrosis factor-a synthesis inhibitor, 3,6'-dithiothalidomide, reverses behavioral impairments induced by minimal traumatic brain injury in mice. J Neurochem [Internet]. 2011 Sep;118(6):1032-42. Available from: http://www.ncbi.nlm.nih.gov/pubmed/21740439.

29. Pan W, Kastin AJ, Rigai T, McLay R, Pick CG. Increased hippocampal uptake of tumor necrosis factor alpha and behavioral changes in mice. Exp brain Res. 2003 Mar;149(2):195-9.

30. Baratz R, Tweedie D, Wang J-Y, Rubovitch V, Luo W, Hoffer BJ, et al. Transiently lowering tumor necrosis factor-a synthesis ameliorates neuronal cell loss and cognitive impairments induced by minimal traumatic brain injury in mice. J Neuroinflammation [Internet]. 2015 Mar 7;12:45. Available from: http://www.ncbi.nlm.nih.gov/pubmed/25879458.

31. Helenius TO, Misiorek JO, Nyström JH, Fortelius LE, Habtezion A, Liao J, et al. Keratin 8 absence down-regulates colonocyte HMGCS2 and modulates colonic ketogenesis and energy metabolism. Mol Biol Cell. 2015 Jun;26(12):2298-310.

32. De Feyter HM, Behar KL, Rao JU, Madden-Hennessey K, Ip KL, Hyder F, et al. A ketogenic diet increases transport and oxidation of ketone bodies in RG2 and $9 \mathrm{~L}$ gliomas without affecting tumor growth. Neuro Oncol. 2016;18(8):1079-87.

33. Dhandapani PK, Lyyski AM, Paulin L, Khan NA, Suomalainen A, Auvinen P, et al. Phenotypic effects of dietary stress in combination with a respiratory chain bypass in mice. Physiol Rep. 2019;7(13):e14159.

34. Griffith CM, Macklin LN, Bartke A, Patrylo PR. Differential Fasting Plasma Glucose and Ketone Body Levels in GHRKO versus 3xTg-AD Mice: A Potential Contributor to Aging-Related Cognitive Status? Int J Endocrinol. 2017;2017:9684061.

35. Rodgers RJ, Dalvi A. Anxiety, defence and the elevated plus-maze. Neurosci Biobehav Rev [Internet]. 1997 Nov;21(6):801-10. Available from: http://www.ncbi.nlm.nih.gov/pubmed/9415905.

36. Fernández Espejo E. Structure of the mouse behaviour on the elevated plus-maze test of anxiety. Behav Brain Res [Internet]. 1997 Jun;86(1):105-12. Available from: http://www.ncbi.nlm.nih.gov/pubmed/9105588.

37. Bevins RA, Besheer J. Object recognition in rats and mice: a one-trial non-matching-to-sample learning task to study "recognition memory". Nat Protoc. 2006;1(3):1306-11.

38. Dix SL, Aggleton JP. Extending the spontaneous preference test of recognition: evidence of objectlocation and object-context recognition. Behav Brain Res [Internet]. 1999 Mar;99(2):191-200. Available from: http://www.ncbi.nlm.nih.gov/pubmed/10512585.

39. Baratz R, Rubovitch V, Frenk H, Pick CG. The influence of alcohol on behavioral recovery after mTBI in mice. J Neurotrauma [Internet]. 2010 Mar;27(3):555-63. Available from: http://www.ncbi.nlm.nih.gov/pubmed/20001584. 
40. Morrison HW, Filosa JA. Sex differences in astrocyte and microglia responses immediately following middle cerebral artery occlusion in adult mice. Neuroscience. 2016.

41. Rubovitch V, Pharayra A, Har-Even M, Dvir O, Mattson MP, Pick CG. Dietary Energy Restriction Ameliorates Cognitive Impairment in a Mouse Model of Traumatic Brain Injury. J Mol Neurosci. 2019 Apr;67(4):613-21.

42. Hyder AA, Wunderlich CA, Puvanachandra P, Gururaj G, Kobusingye OC. The impact of traumatic brain injuries: A global perspective. NeuroRehabilitation. 2007.

43. Tamargo IA, Bader M, Li Y, Yu SJ, Wang Y, Talbot K, et al. Novel GLP-1R/GIPR co-agonist "twincretin" is neuroprotective in cell and rodent models of mild traumatic brain injury. Exp Neurol. 2017.

44. Prins ML, Fujima LS, Hovda DA. Age-dependent reduction of cortical contusion volume by ketones after traumatic brain injury. J Neurosci Res. 2005 Nov;82(3):413-20.

45. Stamatovic SM, Martinez-Revollar G, Hu A, Choi J, Keep RF, Andjelkovic AV. Decline in Sirtuin-1 expression and activity plays a critical role in blood-brain barrier permeability in aging. Neurobiol Dis. 2019;126:105-16.

46. Zhu H, Wang Z yuan, Zhu X, Wu X, xin, Li E, Xu Y. Icariin protects against brain injury by enhancing SIRT1-dependent PGC-1a Expression in experimental stroke. Neuropharmacology. 2010.

47. Moreno CL, Mobbs CV. Epigenetic mechanisms underlying lifespan and age-related effects of dietary restriction and the ketogenic diet. Mol Cell Endocrinol. 2017 Nov;455:33-40.

48. Donmez G, Arun A, Chung CY, Mclean PJ, Lindquist S, Guarente L. SIRT1 protects against a-synuclein aggregation by activating molecular chaperones. J Neurosci. 2012.

49. Qubty D, Rubovitch V, Benromano T, Ovadia M, Pick CG. Orally Administered Cinnamon Extract Attenuates Cognitive and Neuronal Deficits Following Traumatic Brain Injury. J Mol Neurosci. 2021.

50. Mu J, Wang T, Li M, Guan T, Guo Y, Zhang X, et al. Ketogenic diet protects myelin and axons in diffuse axonal injury. Nutr Neurosci. 2021.

51. Mcdowell S, Whyte J, D’Esposito M. Working memory impairments in traumatic brain injury: Evidence from a dual-task paradigm. Neuropsychologia. 1997.

52. Stuss DT, Ely P, Hugenholtz H, Richard MT, LaRochelle S, Poirier CA, et al. Subtle neuropsychological deficits in patients with good recovery after closed head injury. Neurosurgery. 1985.

\section{Figures}




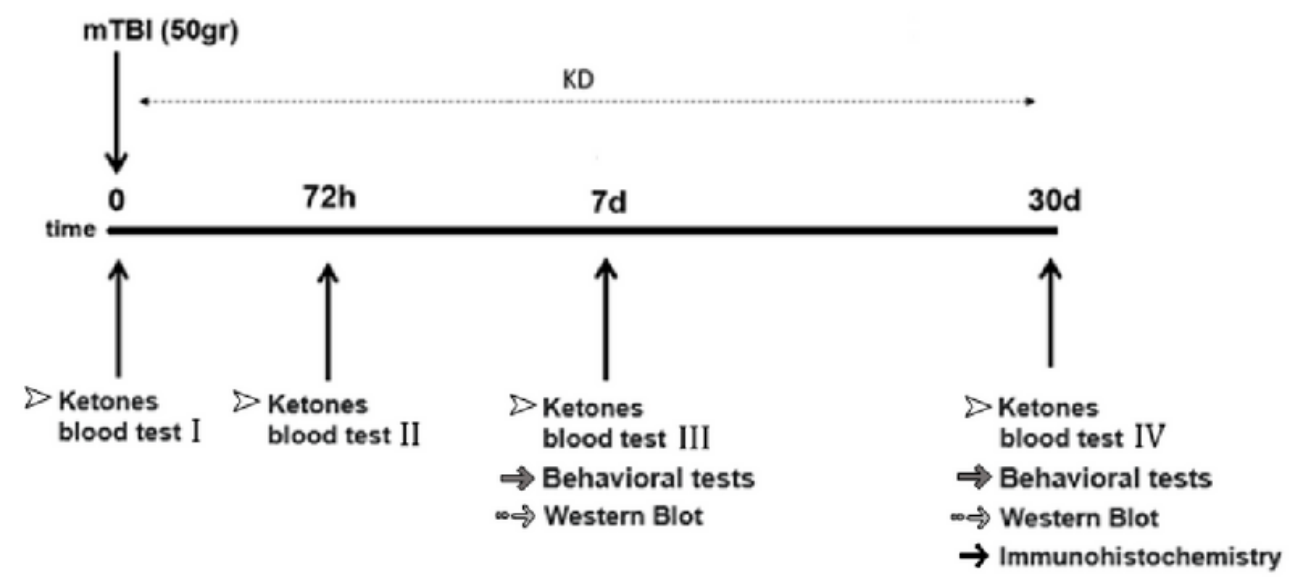

Figure 1

\section{Figure 1}

Study timeline. Animals were exposed to mTBI or sham and checked for ketone bodies in the blood at days $0,3,7$ and 30 . Mice were then fed $\mathrm{KD} / \mathrm{SD}$ for 3, 7, or 30 days. Behavioral tests to assess behavior and cognitive abilities were carried out at 7 and 30 days following $\mathrm{mTBI}$ in separate cohorts. Western blot analysis to assess changes in SIRT1 levels following mTBI and diets management was performed at 7 and 30 days post-mTBI. Immunohistochemical staining to evaluate neurodegeneration and neuroinflammation was performed at 30 days following the mTBI challenge. 


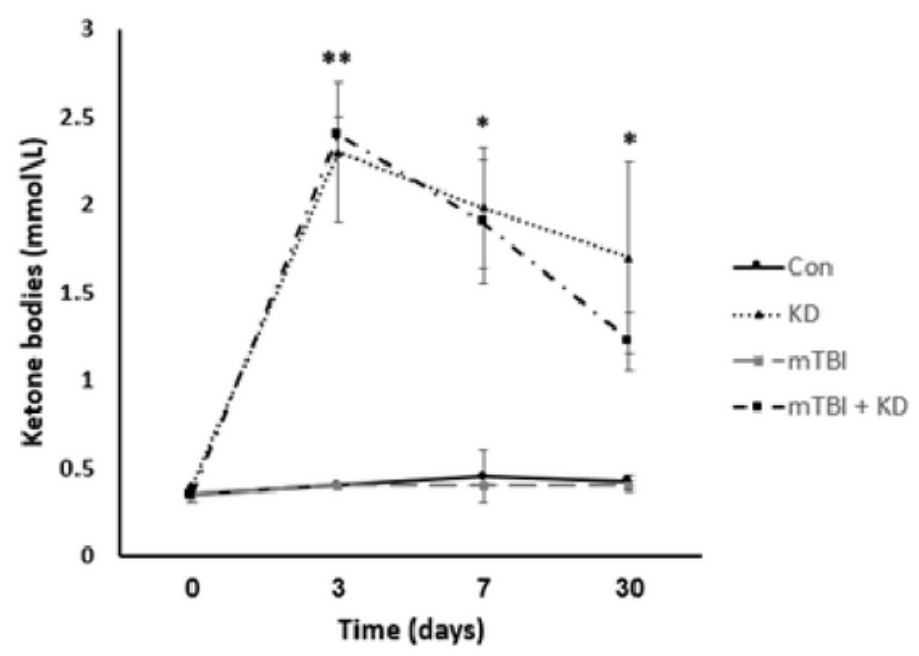

Figure 2

\section{Figure 2}

Ketone body levels in blood. Mice that received KD (with or without $\mathrm{mTBI}$ ) demonstrated a prominent increase in ketone bodies compared to mice fed SD at 3, 7, and 30 days after the diet was initiated. Oneway ANOVA revealed a significant difference at 3 days $[F(3,4)=29.9 ; * \star p<0.01]$, at 7 days $[F(3,9)=4.40$; $\left.{ }^{*} p<0.05\right]$ and at 30 days $\left[F(3,18)=6.20 ;{ }^{*} p<0.05\right](n=3-5)$. 

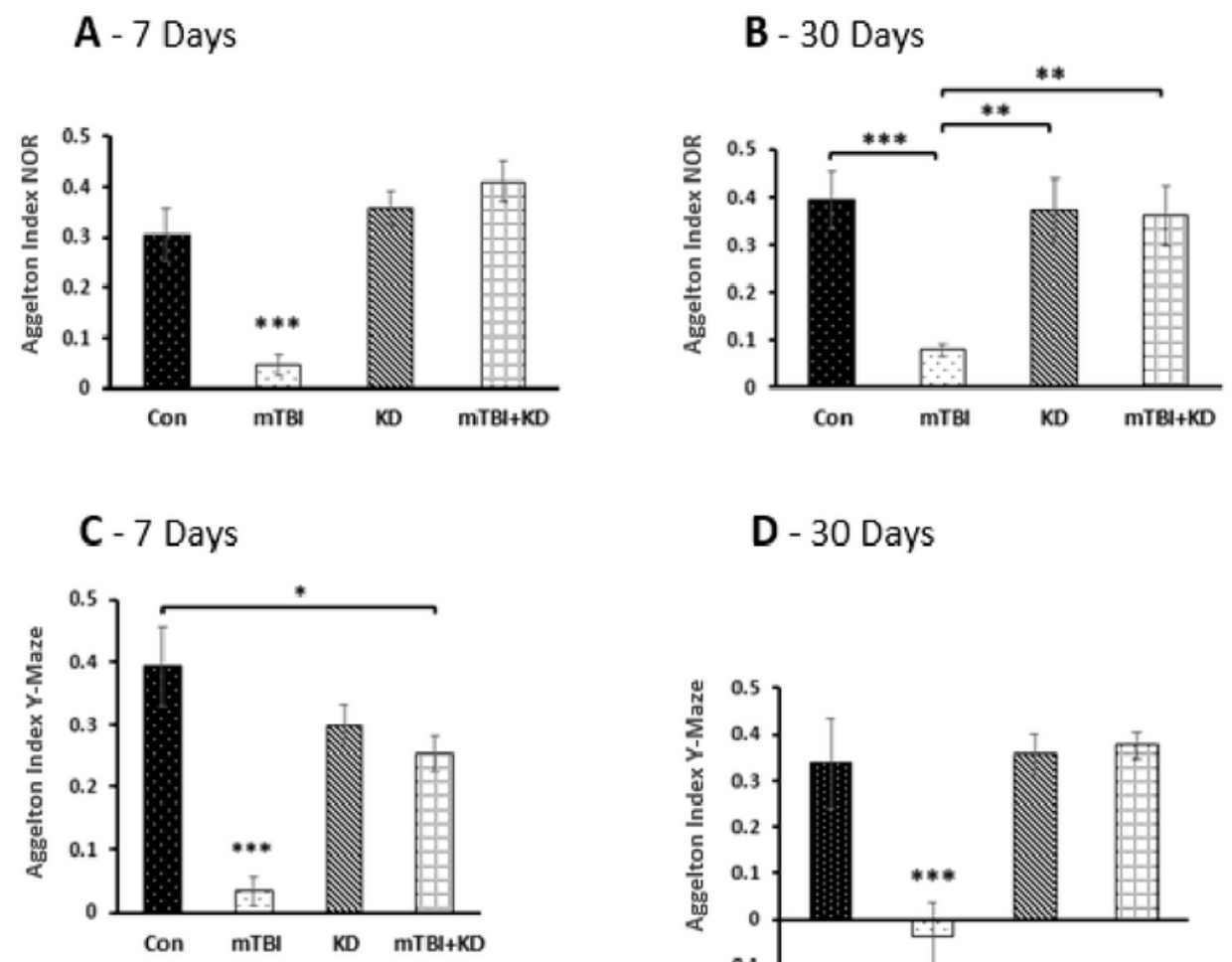

$$
\text { D - } 30 \text { Days }
$$

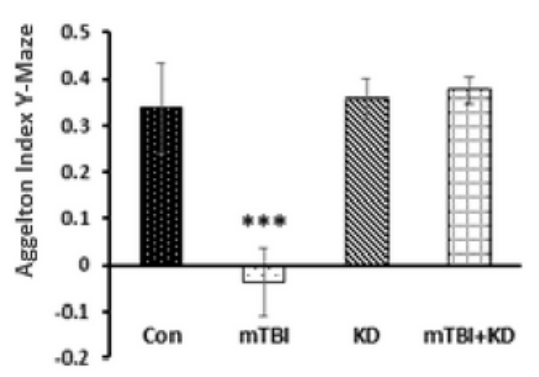

Figure 3

\section{Figure 3}

Ketogenic diet leads to improvement in mTBI-induced cognitive impairments at 7 and 30 days post-mTBI. Visual memory was assessed using the novel object recognition (NOR) paradigm at 7 days (A) and 30 days (B) post-injury. Aggelton index represents the relative time that animals spend exploring a novel object compared to a familiar one, which reflects visual memory. One-way ANOVA test followed by Fisher's LSD post hoc analysis revealed that the preference index of $\mathrm{mTBI}$ group was significantly lower than all other groups at both time points $[A: F(3,41)=12.3 ; * \star \star p<0.001$ and $B: F(3,34)=8.50$; $* \star * p>$ 0.001]. Spatial memory was evaluated using the Y-maze paradigm at 7 (C) and 30 days (D) post-injury. Aggelton index was used to represent the relative time that animals spent exploring a maze's novel arm compared to a familiar one, reflecting spatial memory. One-way ANOVA followed by Fisher's LSD post hoc analysis revealed that the preference index of $\mathrm{mTBI}$ group was significantly lower than all other groups at both time points [C: $F(3,45)=14.5 ; * \star * p<0.001$ and $D$ : $F(3,36)=7.35 ; * \star * p<0.001, * \star p<0.01](n=8-16)$. 
A - Right Cortex

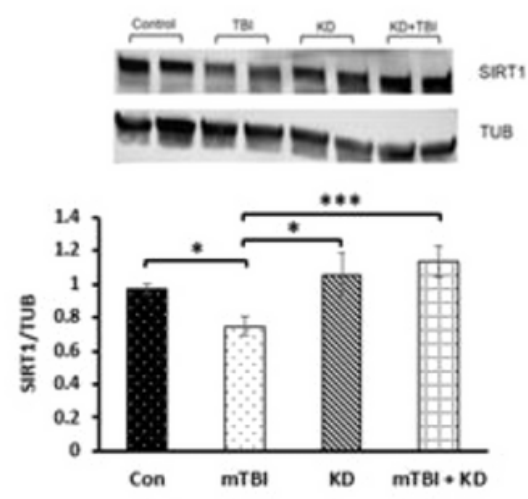

C - Right Hippocampus

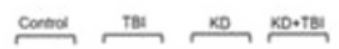

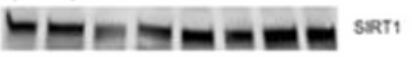

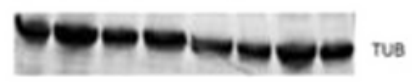

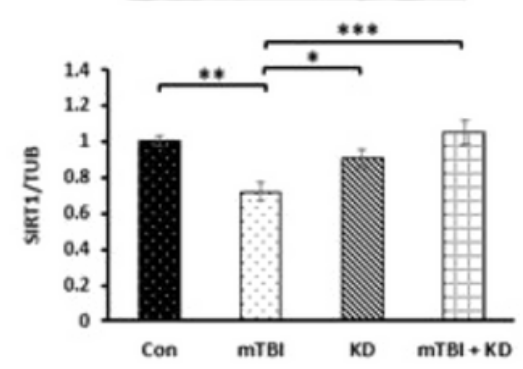

B - Left Cortex
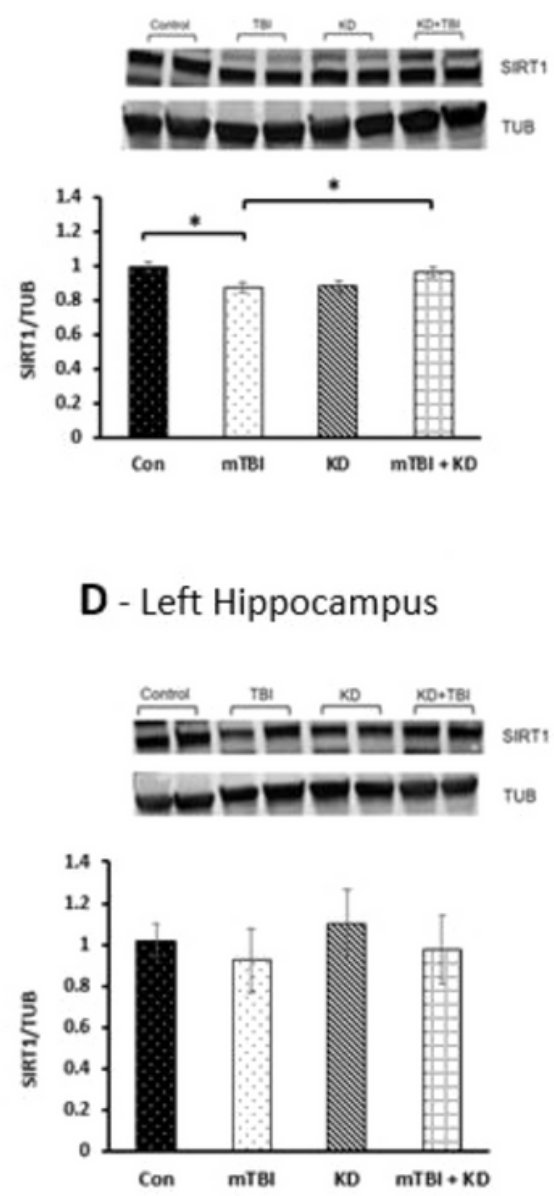

Figure 4

\section{Figure 4}

Effect of KD on SIRT1 expression in the cortex and the hippocampus of mTBI mice. (A) The levels of SIRT1 were significantly reduced 30 days post-injury in the right cortex of mTBI mice compared with sham mice. KD prevented this reduction. One-way ANOVA revealed a significant elevation in expression SIRT1 in mTBI+KD. [F(3,20) $\left.=4.9 ;{ }^{* *} p<0.01,{ }^{*} p<0.05\right]$. (B) KD induced a similar protective effect in the left cortex; the levels of SIRT1 were significantly higher compared with the injured mice. One-way ANOVA revealed a significant elevation in expression SIRT1 in $\mathrm{mTBI}+\mathrm{KD}\left[\mathrm{F}(3,16)=3.36,{ }^{*} \mathrm{p}<0.05\right]$. (C) The levels of SIRT1 have significantly reduced 30 days post-injury in the right hippocampus of mTBI mice compared with control. KD prevented this reduction. One-way ANOVA revealed a significant elevation in expression 
SIRT1 in mTBI+KD. [F(3,17) = 8.74; $\left.{ }^{\star \star \star} p<0.001^{\star \star} p<0.01,{ }^{\star} p<0.05\right]$. (D) SIRT1 levels in post-injury mice were not changed in the left hippocampus compared with other groups. One-way ANOVA revealed no differences in expression SIRT1 between all groups. $[F(3,15)=2.27$, NS $=0.848](n=4-7)$.
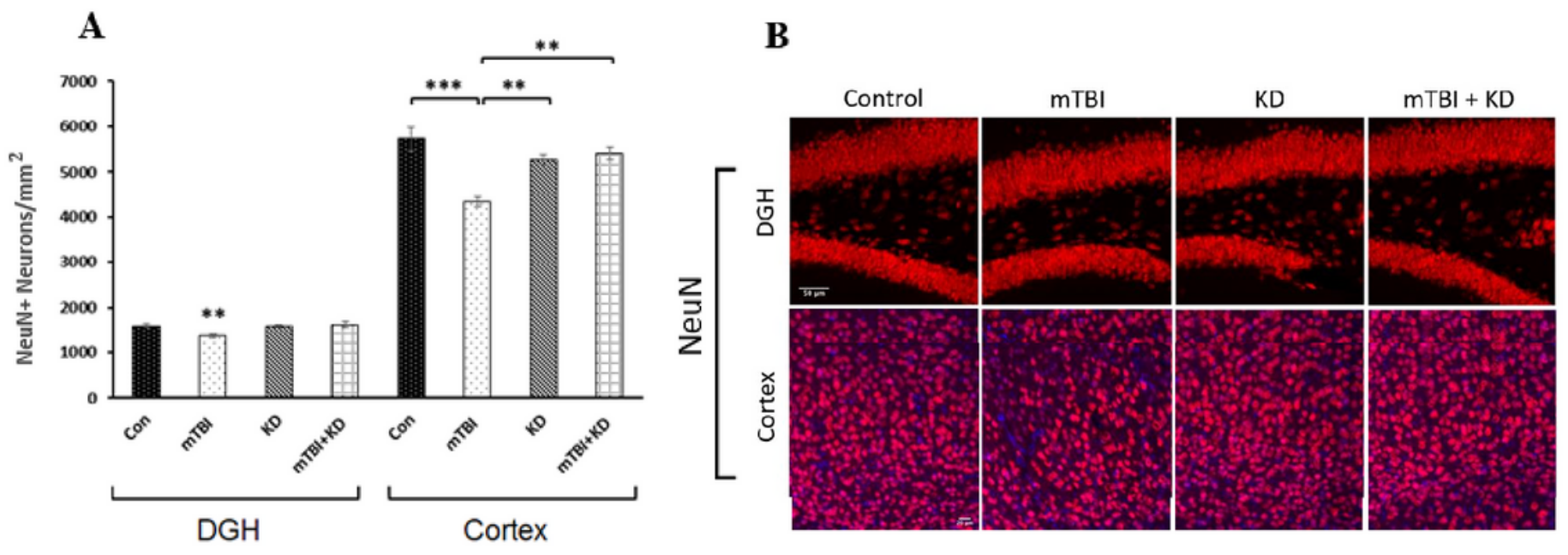

Figure 5

\section{Figure 5}

Ketogenic Diet management elevates mTBI-induced neuronal survival, 30 days post-injury. (A) Two-way ANOVA test followed by Fisher's LSD post hoc analysis demonstrated that mTBI induction led to a significant decrease in the density of $\mathrm{NeuN}+$ neurons compared to sham tissues in all regions tested [DGH: $F(3,15)=7.72 ;{ }^{* \star} p<0.01$, Cortex: $\left.F(3,13)=12.06 ;{ }^{* \star *} p<0.001\right]$. With $K D$, there were significantly greater numbers of NeuN+ neurons than in the untreated mTBI mice $\left({ }^{* *} p<0.01\right)$, suggesting an increase in neuronal survival following the injury. (B) Representative images of immunohistochemical staining in the DGH and the temporal cortex are presented. NeuN positive cells are shown in red, and nuclei in blue; the scale bars are $50 \mu \mathrm{m}$ in the DGH and $20 \mu \mathrm{m}$ in the temporal cortex $(n=3-5)$. 

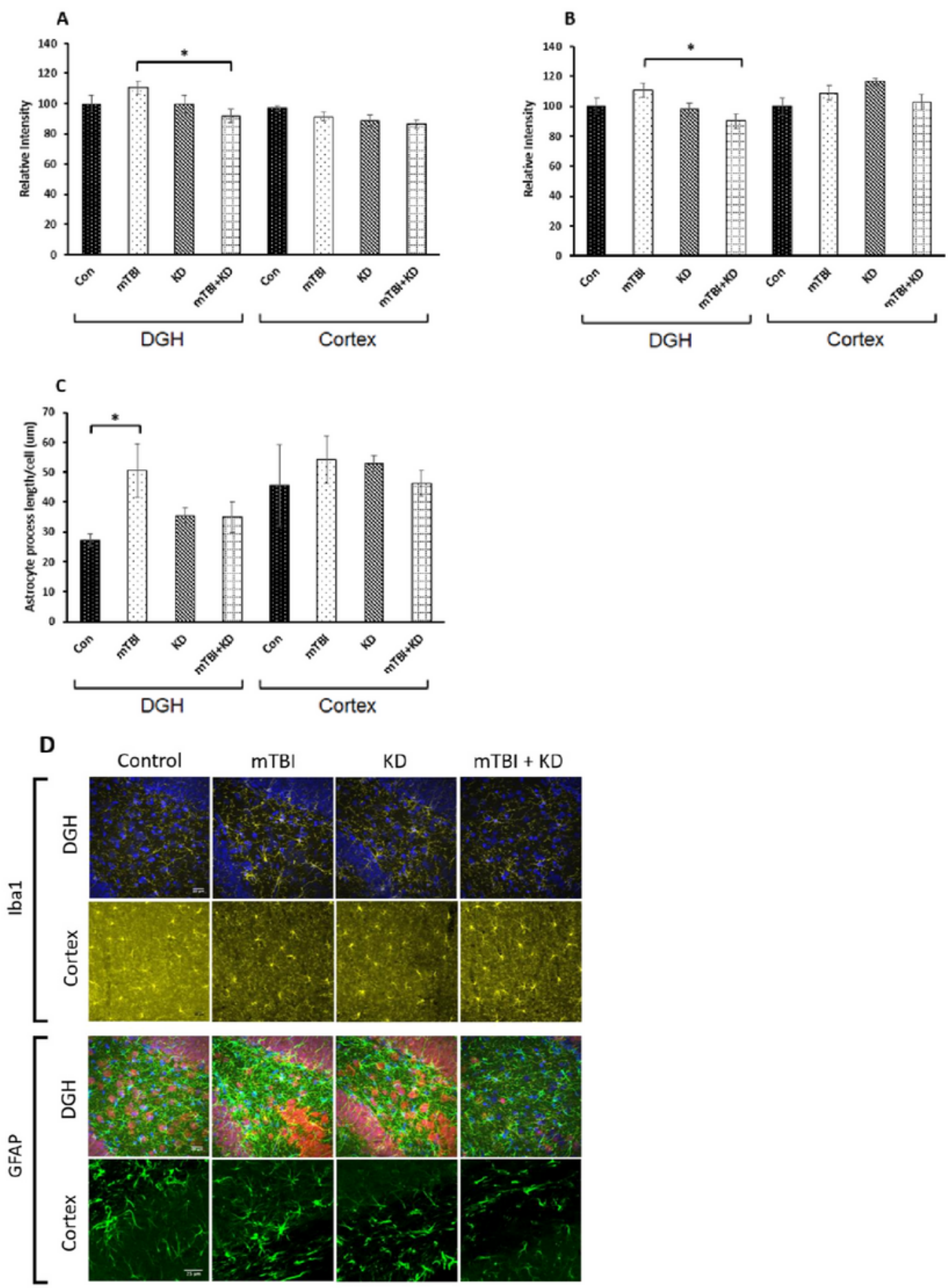

Figure 6

\section{Figure 6}

$\mathrm{KD}$ reduces the $\mathrm{mTBl}$-induced elevation in activated microglia expression and reactive astrocyte expression in the DGH but not in the temporal cortex at 30 days post-injury. Graphs present quantification of the total surface labeled with (A) Iba-1 and (B) GFAP in the DGH and the temporal cortex. Two-way ANOVA followed by Fisher's LSD post hoc analysis revealed that mTBI induction resulted in a nonsignificant elevation in Iba-1 and GFAP expressing cells compared to sham tissues in the regions tested 
[Iba1: $F(7,44)=6.91$; GFAP: $F(7,28)=10.98]$. KD following mTBI prevented the elevation in Iba1 and GFAP expression compared to mTBI mice in the DGH and the cortex $(p<0.05)$. In addition, (C) Astrocyte morphology in the DG Hilus region displayed approximately twice the ramification as the control samples $[F(7,28)=5.88 ; p<0.05]$. (D) Representative images of immunohistochemical staining in the DGH and the temporal cortex are presented. Iba-1 positive cells are shown in yellow, GFAP positive cells are shown in green, and NeuN+ cells in red. The scale bars are 20-25 $\mu \mathrm{m}(n=4-5)$. 